\title{
A MULTI-OBJECTIVE APPROACH FOR DETERMINING THE NUMBER OF BLADES ON A NACA MARINE PROPELLER
}

\author{
UDC 629.5(05) 629.5.035:629.5.024.71
}

Original scientific paper

\begin{abstract}
Summary
This paper numerically discusses how the performance of a NACA marine propeller is affected by the number of blades, which is one of the most crucial geometrical parameters determining the performance of a propeller. Results are presented in terms of the hydrodynamic and structural parameters. The results show that changing the number of blades changes the hydrodynamic efficiency, torque, thrust, cavitation behaviour and structural stiffness of the propeller nonlinearly. Furthermore, it is shown that the propellers structural lifetime is shortened by increasing the number of blades. Hence, the propeller's number of blades is a multi-objective function and will be discussed in this research. The applied tool which is used to study the hydrodynamic performance of the propellers is a RANS-based CFD one and the FEM is considered to study the structural behaviour of the propellers.
\end{abstract}

\section{Keywords: $\quad$ marine propeller; number of blades; CFD; FEM; multi-objective function}

\section{Introduction}

The geometry of a marine propeller is very complicated because of the numerous geometrical parameters involved, such as number of blades, diameter, pitch ratio, and camber and thickness distributions. The designer must adjust these parameters so that the propeller produces the required thrust and has the minimum possibility of cavitation occurring, acceptable efficiency, low vibration, and high structural lifetime. Each parameter has specific effects on the designer's objectives and must be carefully adjusted. For example, increasing the pitch ratio of a propeller augments the thrust produced by the propeller and would nonlinearly change its hydrodynamic efficiency. Furthermore, changing the pitch ratio of the blades of a propeller alters the angles of the blades relative to the axis of rotation and affects their structural behaviour. Therefore, its increment would reinforce the blades against the bending in the axial direction.

The developments which have occurred in computational resources have made researchers use numerical methods in order to accurately analyze the flow fields around complicated devices. Therefore, the hydrodynamics researchers have been interested in simulating the flow around the propellers by means of RANS-based Computational Fluid Dynamics (CFD) methods for the last decade. Several research projects have been performed 
on marine propellers in open water or wake conditions using numerical methods [1-8]. For example, Ji et al. (2011) [6] performed an unsteady numerical simulation of cavitating turbulent flow around a highly skewed marine propeller. They used the commercial code CFX based on Reynolds-averaged Navier-Stokes (RANS) equation with SST-k- $\omega$ turbulence model. The maximum difference of pressure fluctuation between the calculation and experiment reached $20 \%$ in this study. Peng et al. (2013) [7] investigated the effects of turbulence models on predicting the performance of a specific marine propeller in the open water condition. They showed that eddy-viscosity turbulence models predicted the hydrodynamic coefficients of the propeller more accurately than Reynolds-stress models. Furthermore, they concluded that all of the eddy-viscosity turbulence models predicted about the same performance for the propeller. In spite of the extensive research done on predicting the performance of propellers, the effects of geometrical parameters on the performance of propellers have rarely been discussed. For example, skew and rake are among the typical investigated parameters by the researchers in recent years $[1,3,5]$.

One of the most important geometrical parameters of a propeller is the number of blades. This parameter is related to the propeller's geometrical configuration, the occurrence of cavitation, the extent of deflection of the blades (vibrational behaviour), the propeller's structural lifetime, and its hydrodynamic efficiency. The importance of the subject is revealed in the design procedure of a marine propeller using a specific mass and a specific expanded area ratio. Note that this ratio is estimated for enabling the propeller overcomes the cavitation occurrence. Hence, the effects of the division number of the mentioned properties which is indicative of how the number of blades affects the performance of the propeller should be discussed before starting the design process. The designer has to exactly know what happens if he increases or decreases the number of blades even one. As mentioned before, the determination procedure of the number of blades of a propeller is a multi-objective approach, which was the topic of this research. The 4-digit NACA airfoils are selected as the cross sections of the propellers in this research because they could be simply modelled and also provide a good comparability between the results of the research. Furthermore the 4-digit NACA airfoils have acceptable similarities to the applied cross sections in the geometries of common propellers. Moreover, the overall shape of the blades is determined similarly to the basic shape of the un-skewed marine propellers before modifying their edges, especially in the outer radius ratios. In this way, the considered propellers in this research are similar to common marine ones and the obtained results of this research are reliable in the case of such propellers. The obtained results would help the designers to make accurate decisions on determining the number of blades on marine propellers.

In this research, a finite volume RANS-based CFD tool is used to predict the hydrodynamic performance of the propellers in the steady state way. Furthermore, a FEM tool is used to predict their structural behaviour. The hydrodynamic analyses of the propellers are performed and the obtained pressure distributions around the propellers are used in FEM models to evaluate the structural stresses on the blades and the resulting deformations.

\section{Numerical simulations}

\subsection{Computational Fluid Dynamics}

The governing equations for analysing the flow fields are consisted of momentum, mass, and energy conservation equations. In this research, since isothermal flow is assumed, the energy conservation equation is not needed. Through applying the Reynolds averaging method on the governing equations of an incompressible Newtonian fluid without any external forces, the governing equations can be stated as follows: 


$$
\begin{aligned}
& \frac{\partial \overline{u_{i}}}{\partial x_{i}}=0 \\
& \frac{\partial\left(\rho \overline{u_{i}}\right)}{\partial t}+\frac{\partial}{\partial x_{j}}\left(\rho \overline{u_{i}} \overline{u_{j}}\right)=-\frac{\partial(\bar{p})}{\partial x_{i}}+\mu \frac{\partial}{\partial x_{j}}\left(\frac{\partial \overline{u_{i}}}{\partial x_{j}}+\frac{\partial \overline{u_{j}}}{\partial x_{i}}\right)+\frac{\partial}{\partial x_{j}}\left(-\rho \overline{u_{i}^{\prime} u_{j}^{\prime}}\right)
\end{aligned}
$$

Where $u_{i}{ }^{\prime}$ are the fluctuating parts of the velocity components, $\overline{u_{i}}$ are the mean values of the velocity components, and $\tau_{i j}=\rho \overline{u_{i}^{\prime} u_{j}^{\prime}}$ is Reynolds stresses tensor. Reynolds stresses could not be obtained from the mentioned governing equations, so some additional equations are needed. Applying Boussinesq's eddy-viscosity assumption, these terms are related to the mean velocity gradients and the turbulent Eddy viscosity and could be stated for the incompressible flow as follows:

$$
\tau_{i j}=\mu_{t}\left[\frac{\partial \overline{u_{i}}}{\partial x_{j}}+\frac{\partial \overline{u_{j}}}{\partial x_{i}}\right]
$$

Where $\mu_{t}$ is the turbulent Eddy viscosity. The turbulent viscosity of the flow could be obtained by means of turbulence models. The most famous turbulence models are two-equation models, i.e., k- $\varepsilon$ and $\mathrm{k}-\omega$ models, because they are relatively simple, and they are accurate in numerous types of flow fields. The $\mathrm{RNG}^{1}-\mathrm{k}-\varepsilon$ turbulence model is used in this research because it is more responsive to rotating flows and streamlines' curvatures [9]. The RNG-k- $\varepsilon$ turbulence model's governing equations are as follows [10]:

$$
\begin{aligned}
& \overline{u_{j}} \frac{\partial k}{\partial x_{j}}=\frac{1}{\rho} \frac{\partial}{\partial x_{j}}\left[\left(\mu+\frac{\mu_{t}}{\sigma_{k}}\right) \frac{\partial k}{\partial x_{j}}\right]+P_{k}-\varepsilon \\
& \overline{u_{j}} \frac{\partial \varepsilon}{\partial x_{i}}=\frac{1}{\rho} \frac{\partial}{\partial x_{j}}\left[\left(\mu+\frac{\mu_{t}}{\sigma_{\varepsilon}}\right) \frac{\partial \varepsilon}{\partial x_{j}}\right]+C_{1 \varepsilon} \frac{\varepsilon}{k} P_{k}-C_{2 \varepsilon} \frac{\varepsilon^{2}}{k} \\
& P_{k}=v_{t}\left(\frac{\partial \overline{u_{i}}}{\partial x_{j}}+\frac{\partial \overline{u_{j}}}{\partial x_{i}}\right) \frac{\partial \overline{u_{i}}}{\partial x_{j}} \\
& \mu_{t}=\rho C_{\mu} \frac{k^{2}}{\varepsilon}=\rho v_{t}
\end{aligned}
$$

Where $k$ is the turbulent kinetic energy, $\varepsilon$ is the turbulent kinetic energy dissipation rate and $p_{k}$ is the turbulent kinetic energy production term. In equation (3), $C_{\mu}, C_{1 \varepsilon}, C_{2 \varepsilon}, \sigma_{k}, \sigma_{\varepsilon}$ are constants.

The method which is used to model the rotational motion of the propeller is the moving reference frame one. Thus, the rotational motion is applied to the computational domain with the same rotational speed as the propeller, but in the opposite direction. Therefore, the velocity vector is changed from the absolute form to the relative form according to $\mathbf{v}=\mathbf{v}_{r}+\boldsymbol{\omega} \times \mathbf{r}$, where $\mathbf{r}$ is the position vector relative to the origin and $\boldsymbol{\omega}$ is the rotational velocity vector, and the governing equations are solved in the relative form. This approach is the simplest technique for modelling the problems with rotary boundary conditions, while the flow which is entering

\footnotetext{
${ }^{1}$ Re-Normalization Group
} 
to the rotary part is uniform relative to the axis of rotation, in a steady state way and has been applied by many other researchers [4-8]. Hence, this technique is used in this research.

A finite-volume, RANS-based CFD code is used in this research. The pressure-velocity coupling method is SIMPLEC ${ }^{2}$ [11], the pressure discretization method is PRESTO ${ }^{3}$, and the discretization method used for convective terms is QUICK ${ }^{4}[12]$.

2.2 Structural analysis

The governing equations of the applied FEM code are as follows:

$$
M \ddot{u}+C \dot{u}+K u=F
$$

Where $[M],[C]$ and $[K]$ are mass, damping, and stiffness matrices respectively, and $\ddot{u}, \dot{u}$, and $u$ are acceleration, velocity, and displacement vectors respectively. The applied external forces used in the FEM model are represented as the $F$ vector which includes:

- $\quad$ The pressure force over the propeller blades which is obtained from the CFD solution and is computed as $F_{h}=\int_{A} P d s$.

- The centrifugal force which is computed as $F_{c e}=\int_{v} \rho_{p} r_{e} \omega^{2} d v$ where $r_{e}$ is the radial position of the centre of the element, $\rho_{p}$ is the density of the propeller material, and $\omega$ is the rotational speed of the propeller [13].

2.3 Dimensionless hydrodynamic parameters of the propellers

In order to express the hydrodynamic parameters of a propeller in open water condition, it is common to use the dimensionless parameters shown below:

$$
\begin{array}{lc}
\text { Thrust Coefficient : } & \mathrm{K}_{\mathrm{T}}=\frac{\mathrm{T}}{\rho \mathrm{n}^{2} \mathrm{D}^{4}} \\
\text { Torque Coefficient : } & \mathrm{K}_{\mathrm{Q}}=\frac{\mathrm{Q}}{\rho \mathrm{n}^{2} \mathrm{D}^{5}} \\
\text { Efficiency : } & \eta=\frac{\mathrm{TV}_{\mathrm{a}}}{2 \pi \mathrm{nQ}} \\
\text { Advance Coefficient : } & \mathrm{J}=\frac{\mathrm{V}_{\mathrm{a}}}{\mathrm{nD}}
\end{array}
$$

where $T$ is the propeller's produced thrust in Newton $(\mathrm{N}), Q$ is the axial torque in Newton-meter (N.m), $V_{a}$ is the advance velocity in meters per second $(\mathrm{m} / \mathrm{s}), n$ is the revolutions per second (rps), $D$ is the diameter in meters (m), and $\rho$ is the density in kilograms per cubic meter ( $\left.\mathrm{kg} / \mathrm{m}^{3}\right)$ [14].

\footnotetext{
${ }^{2}$ Semi-Implicit method for pressure linked equations-consistent

${ }^{3}$ Pressure staggering option

${ }^{4}$ Quadratic upstream interpolation for convective kinematics
} 


\subsection{Computational Domain}

In order to solve the governing equations of the flow field around the propeller, a computational domain must be considered around the propeller in such a way that the dimensions of the domain do not affect the simulation results. Figure 1 shows that the considered computational domain has had a cylindrical shape.

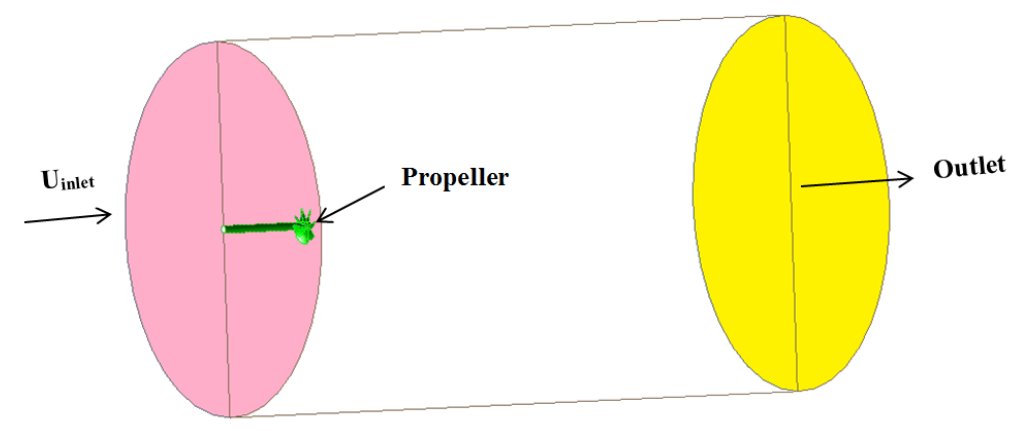

Figure 1: The computational domain

The applied boundary conditions are:

- Velocity inlet at the left end of the domain in Figure 1.

- Pressure outlet at the right end of the domain in Figure 1.

- Velocity inlet on the lateral face of the computational domain in Figure 1.

- Wall boundary condition with zero velocity relative to the domain on the propeller's blades.

Figure 2 shows the dimensions of the considered domain.

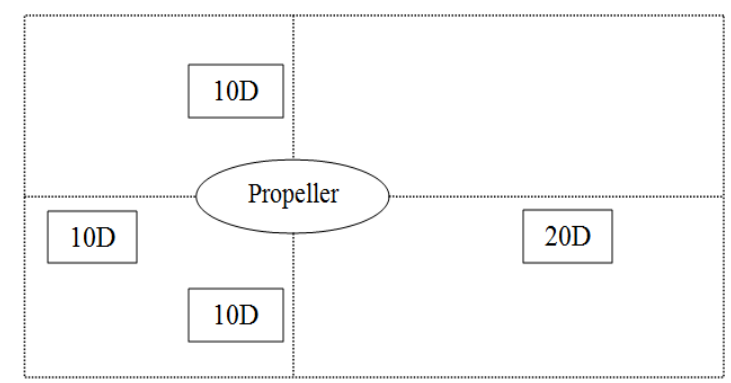

Figure 2: Dimensions of the computational domain (D is the propeller's diameter.)

The dimensions of the computational domain are considered in such a way that the effects of the propeller's revolution on the flow field do not reach the boundaries. In the other words, the flow becomes as uniform as possible near the domain faces. So, the domain must be large enough especially for high blade loadings (high rates of revolution). We performed a study over the different dimensions of the computational domain and tracked the dependency of the produced thrust on the domain's dimensions. The results are reported in Figures $3 \& 4$. It can be concluded that the dependency of the produced thrust on the domain's dimensions would be minimized by using the domain's dimensions reported in Figure 2. 


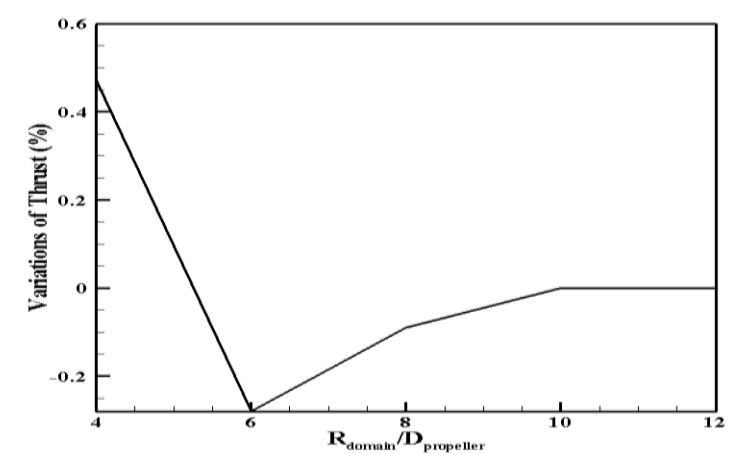

Figure 3: Independency of the CFD results from the cylindrical domain's diameter

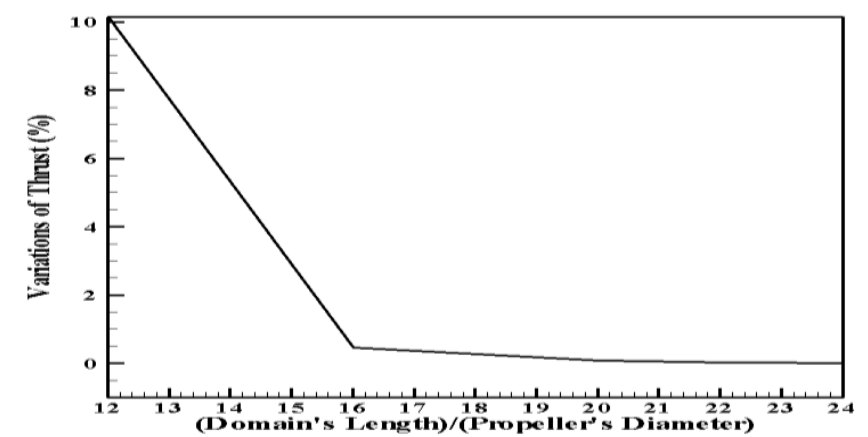

Figure 4: Independency of the CFD results from the cylindrical domain's height downstream the propeller

The mesh is generated in the computational domain in such a way that the hexahedral elements covered the entire domain, except for a small area near the propeller's blades because of some complexities. So, the computational domain is divided into several zones in order to create a hexahedral dominant mesh in all zones, except for a small cylinder around the propeller in which the tetrahedral elements are generated. Figure 5 shows the generated mesh in the computational domain.

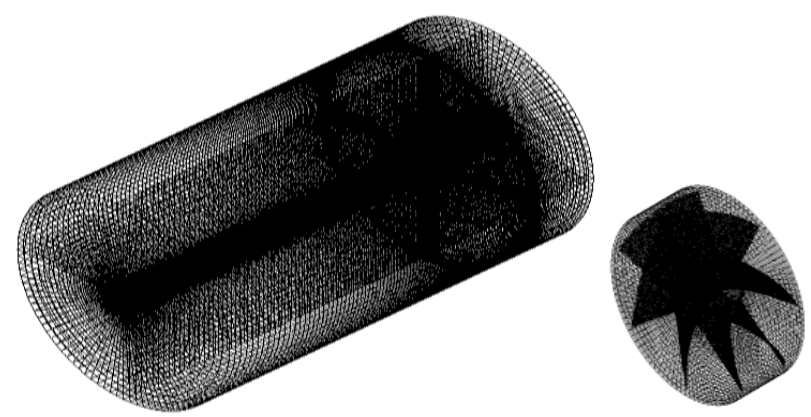

Figure 5: The generated mesh in the computational domain: left) the entire domain; right) around the

propeller

\subsection{Validation}

In order to evaluate the solution's procedure, a standard 6-bladed B-wageningen propeller with a pitch ratio of 1.2 , an expanded area ratio of 1 and a diameter equal to $25 \mathrm{~cm}$ was considered. The dependency of the hydrodynamic efficiency of the selected propeller on the number of elements in the computational domain is studied and the results are presented in Table 1 . The magnitude of error is computed by using equation 9 .

$$
\text { Efficiency error }{ }_{i}=\frac{\text { Efficiency }_{i}-\text { Efficiency }_{i-1}}{\text { Efficiency }_{i-1}} \times 100
$$


Table 1: The effects of the mesh sizing on the propeller's performance

\begin{tabular}{|c|c|c|c|c|c|}
\hline No. & $\begin{array}{c}\text { Element size in } \\
\text { a small cylinder } \\
\text { around the } \\
\text { propeller }(\% \mathrm{D})\end{array}$ & $\begin{array}{c}\text { Blade } \\
\text { surface } \\
\text { mesh } \\
(\% \mathrm{D})\end{array}$ & $\begin{array}{c}\text { Element size } \\
\text { in the zones } \\
\text { far from the } \\
\text { propeller } \\
(\% \mathrm{D})\end{array}$ & $\begin{array}{c}\text { Number } \\
\text { of } \\
\text { Elements } \\
\left(10^{6}\right)\end{array}$ & $\begin{array}{c}\text { Efficiency } \\
\text { Error } \\
(\%)\end{array}$ \\
\hline 1 & 1.5 to 7.5 & 1.5 & 7.5 to 50 & 0.55 & - \\
\hline 2 & 1.5 to 5.0 & 1.5 & 5.0 to 50 & 0.65 & 3 \\
\hline 3 & 1.5 to 3.5 & 1.5 & 3.5 to 50 & 1.20 & 2.6 \\
\hline 4 & 1.5 to 2.5 & 1.5 & 2.5 to 50 & 1.57 & 2.2 \\
\hline 5 & 0.8 & 0.8 & 0.8 to 50 & 12.5 & 2.15 \\
\hline
\end{tabular}

As it is shown in Table 1, by using a mesh sizing equal to 1.5 percent of the propeller's diameter on the propeller blades and in a small cylinder around the propeller a grid consisting of about 1.6 million elements is produced. The dependency of the hydrodynamic efficiency on the number of elements would be minimized by using such grid and the results would be independent from the number of elements.

In order to demonstrate the validity of the obtained simulation procedure, the hydrodynamic coefficients of the considered propeller were extracted. The operational conditions of the propeller were adjusted so that the maximum allowable thrust over the propeller was produced based on Keller's cavitation criterion as follows [14]:

$$
\frac{A_{e}}{A_{0}} \geq \frac{(1.3+0.3 z) T}{D^{2}\left(P_{0}-P_{V}\right)}+K
$$

where $\frac{A_{e}}{A_{0}}$ is the minimum expanded area ratio which makes the propeller able to overcome the cavitation occurrence, $z$ is the propeller's number of blades, $D$ is the propeller's diameter in meters $(\mathrm{m}), P_{0}$ is the summation of the atmospheric and hydrostatic pressure on the propeller's plane in Pascal (pa), $P_{v}$ is the vapor pressure in the ambient temperature in Pascal (pa), $T$ is the propeller's thrust in Newton $(\mathrm{N})$, and $\mathrm{K}$ is the constant which depends to the number of propellers and the type of the vessel and is assumed to be 0.2 is this research, regarding the reference [14]. Based on equation 10, the maximum allowable thrust over the considered propeller was estimated about $1750 \mathrm{~N}$ in an advance coefficient equal to 0.8 in this research. The adjustments of the operational parameters have been performed so that the thrusts which were lower than $1750 \mathrm{~N}$ were produced by the propeller. The maximum error happened in the prediction of the produced thrust and was about $8 \%$ in the considered operational range [15]. The validation diagram is plotted in Figure 6.

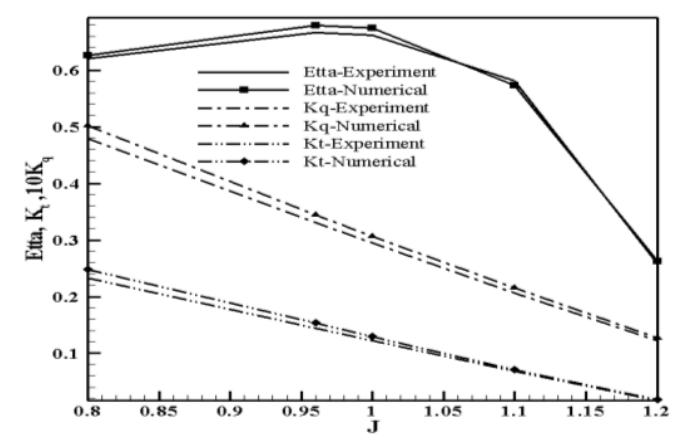

Figure 6: Comparison of the simulation results with the experimental data [15] [15]

So, it was observed that the applied CFD tool which was used with the specified settings has been sufficiently accurate in analysing the flow around the propellers. 
Furthermore, the independency of the FEM results from the number of elements were studied and it was concluded that by using a grid consisting of about 800,000 elements the FEM results would not depend on the number of elements any more. Note that a tetrahedral structural element which was defined by 10 nodes with three degrees of freedom at each one was used in the FEM analyses. This element has had quadratic displacement behaviour. This structural element is well suited for modelling the propeller's structure. Because of the lack of experimental data on the structural behaviour of the considered B-series propeller, the mentioned FEM code was validated by some simple cases such as cantilever beams. It was assumed that the results of the structural analyses of the propellers will be accurate enough if the FEM simulations are precisely performed.

Note that in the present study the simulations are performed in order to compare the results between some different models. So, it is important to have the computational tool which is sophisticated enough from the ranking of the designs perspective and the absolute accuracy of the predictions is not the main concern and it is sufficient to be fairly enough.

\section{Case study}

The main objective of this research is to present a multi-objective determination approach for the number of blades on a single marine propeller. This multi-objective approach includes several important considerations, i.e., preventing cavitation (related to the pressure distribution around the propeller), hydrodynamic efficiency, deflection of the blades (related to the vibrational motion of the blades), maximum Von-Mises stresses at the existing hydrodynamic pressure (related to the structural lifetime), and the axial length of the propeller (related to the physical space of the propeller). In order to perform this research, some propellers with similar geometrical parameters but with different numbers of blades are considered. So, the following assumptions are made:

1 - The diameter is equal to $40 \mathrm{~cm}$.

2- The expanded area ratio is equal to 1.23 and similar shapes are considered for the leading and trailing edges between all models.

3- The pitch ratio is equal to 1.5 .

4- The four-digit NACA cross sections with the location of the maximum thickness equal to $30 \%$ of the length of the chord from the leading edge and with the location of the maximum camber equal to $50 \%$ of the length of the chord from the leading edge are used in all radius ratios.

5 - The camber ratio is equal to $2 \%$ over the blades.

6- A similar linearly-decreasing distribution of the magnitudes of the maximum thickness is used in the radial direction.

7- The numbers of the blades are 3, 4, 5, 6, 7, 8, 9, 11, 13 and 15 in models 1 to 10.

8 - The weight of the propellers is about $4 \mathrm{~kg}$.

In this way, we considered ten different models of the propellers with similar geometrical parameters but with different numbers of blades in order to find the effects of the number of blades on the propeller's performance out. Table 2 provides the radial geometrical properties of ten different models. 
Table 2: Radial properties of the models

\begin{tabular}{|c|c|c|c|c|}
\hline$x(r / R)$ & $\begin{array}{c}\mathrm{t}(\mathrm{mm}) \\
(1 \text { to } 10)\end{array}$ & $\begin{array}{l}f / c(\%) \\
(1 \text { to } 10)\end{array}$ & Cross Section & Number of blades \\
\hline 0.2 & 9.84 & 2 & 4-digit NACA & \multirow{17}{*}{$\begin{array}{c}3,4 \\
5,6 \\
7,8 \\
9,11 \\
13,15\end{array}$} \\
\hline 0.3 & 8.76 & 2 & 4-digit NACA & \\
\hline 0.4 & 7.68 & 2 & 4-digit NACA & \\
\hline 0.5 & 6.6 & 2 & 4-digit NACA & \\
\hline 0.6 & 5.52 & 2 & 4-digit NACA & \\
\hline 0.7 & 4.44 & 2 & 4-digit NACA & \\
\hline 0.75 & 3.9 & 2 & 4-digit NACA & \\
\hline 0.8 & 3.36 & 2 & 4-digit NACA & \\
\hline 0.85 & 2.82 & 2 & 4-digit NACA & \\
\hline 0.9 & 2.28 & 2 & 4-digit NACA & \\
\hline 0.92 & 2.06 & 2 & 4-digit NACA & \\
\hline 0.94 & 1.85 & 2 & 4-digit NACA & \\
\hline 0.96 & 1.63 & 2 & 4-digit NACA & \\
\hline 0.97 & 1.52 & 2 & 4-digit NACA & \\
\hline 0.98 & 1.42 & 2 & 4-digit NACA & \\
\hline 0.99 & 1.3 & 2 & 4-digit NACA & \\
\hline 1 & 1.2 & 2 & 4-digit NACA & \\
\hline
\end{tabular}

where $t$ is the maximum thickness of the cross section, $f$ is the maximum camber of the section, $c$ is the chord length of the section, $r$ is the radial position of the section, and $R$ is the overall radius of the propeller. The material which is used for the structure of the propellers is stainless steel and Table 3 presents its physical properties.

Table 3: Physical properties of stainless steel

\begin{tabular}{|c|c|}
\hline Property & Magnitude \\
\hline Modulus of elasticity $(\mathrm{Pa})$ & $2 \times 10^{11}$ \\
\hline Poisson ratio & 0.3 \\
\hline Density $\left(\mathrm{kg} / \mathrm{m}^{3}\right)$ & 7850 \\
\hline Tensile yield strength $(\mathrm{Pa})$ & $2.5 \times 10^{8}$ \\
\hline Tensile ultimate strength $(\mathrm{Pa})$ & $4.6 \times 10^{8}$ \\
\hline
\end{tabular}

Based on the mentioned assumptions, the weights of all propellers are the same, i.e., about $4 \mathrm{~kg}$ without the hub. One of the created geometries is shown in Figure 7.

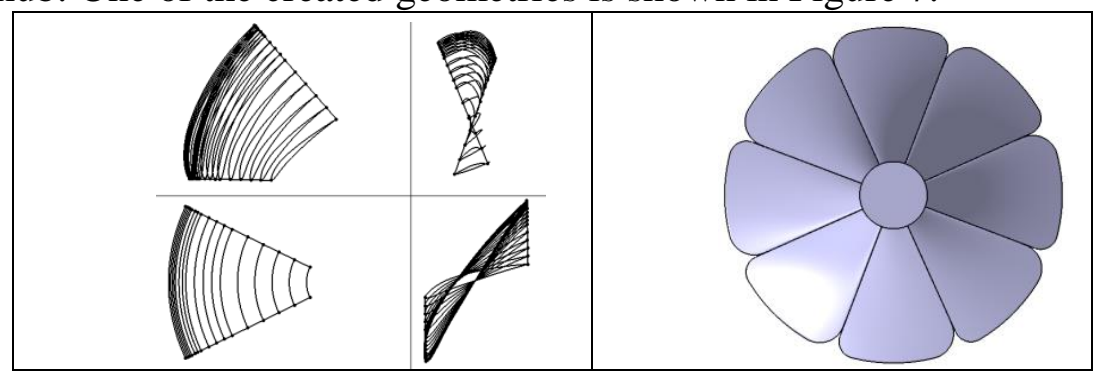

Figure 7: Geometry of the eight-bladed propeller: left) radial cross sections of one blade in the premodelling step; right) the entire propeller 
As shown in Figure 7 and indicated in Table 2, seventeen different radial sections are used in the geometry of each propeller, all of which have four-digit NACA cross sections with similar properties as mentioned earlier. Also note that, as shown in Table 2, in order to keep the weights of the propellers the same, we had to avoid changing the magnitudes of the maximum thickness of the airfoils in the same radius ratios between different models. As the result, we had to increase the number of blades on some propellers that had thicker radial sections relative to their corresponding chord lengths. In the other words, the magnitudes of $t / c(r)_{i}$ are increased by increasing the number of blades. As it is shown in Figure 7, the adopted geometries for the propellers are different from common ones in the outer radius ratios. The reason of selecting such configuration for the propellers was to retain the hydrodynamic load of the blades in their outer parts and make the resultant produced force of the blades further from the roots. So, the bending effect of the thrust would be reinforced and would make a loading condition for the blades which is harder than usual. Therefore, we could have a strict judgment about the propellers from the structural perspective which could increase the reliability of the results in the case of common propellers. In the other hand, since the basic shape of each un-skewed marine propeller would be similar to the presented propeller in Figure 7 (before modifying their leading and trailing edges), the results deemed not to have conflict with the common cases.

\section{Results}

In this research, a velocity of $10 \mathrm{~m} / \mathrm{sec}$ is assumed as the velocity inlet boundary condition of the domain. The rotational speeds of the propellers are changed such that a similar limited range of the advance coefficient is covered between different models.

The extracted hydrodynamic parameters of the propellers with different numbers of blades are compared with each other in Figures 8 to11.

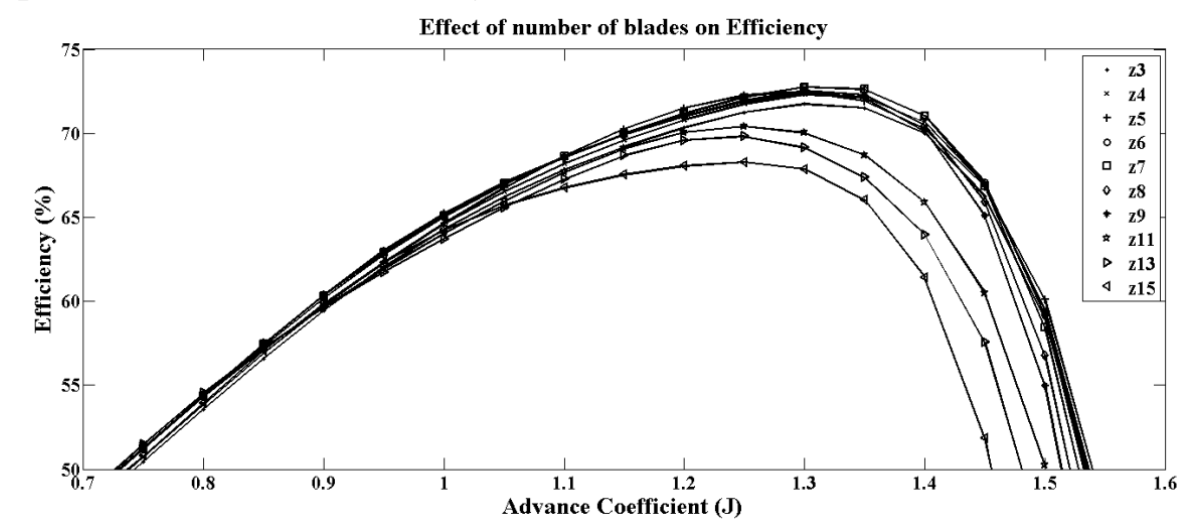

Figure 8: Hydrodynamic efficiency of the propellers versus the number of blades

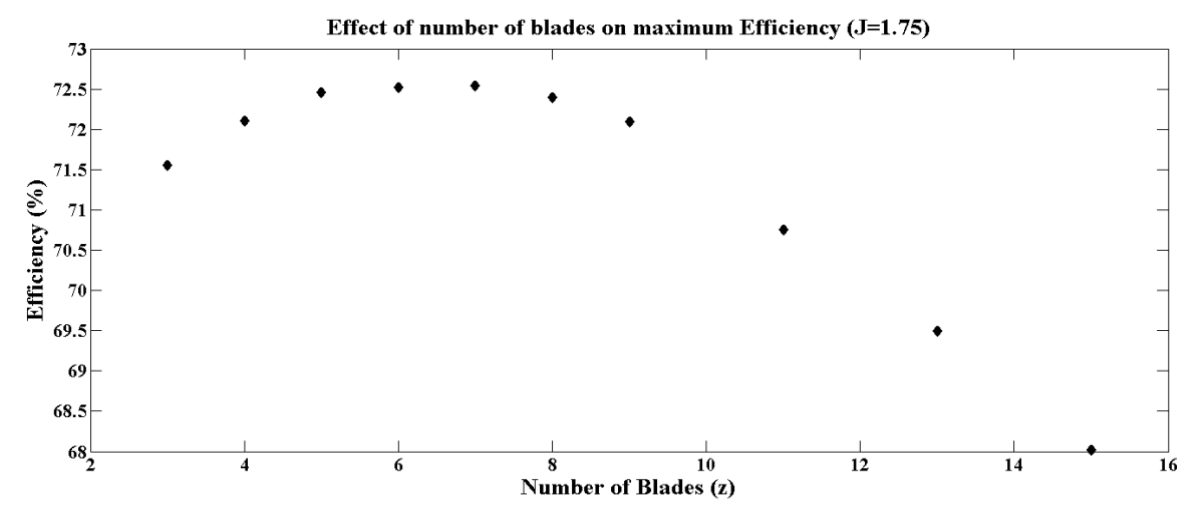

Figure 9: Maximum efficiency of the propellers versus the number of blades 


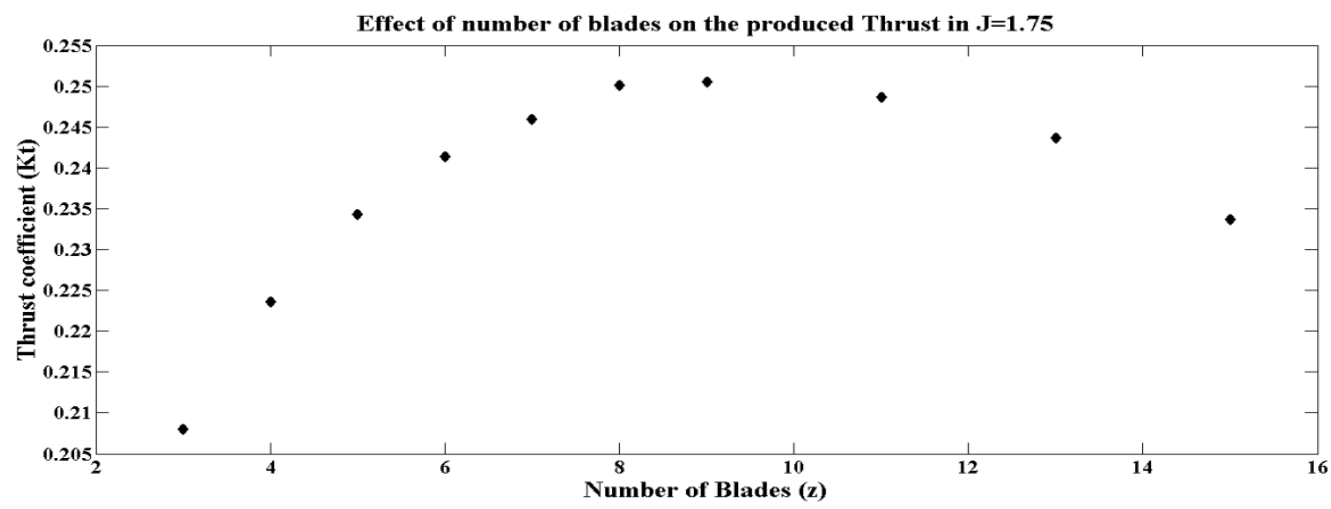

Figure 10: Magnitude of the thrust produced by propellers in the advance coefficient corresponding to the maximum efficiency versus the number of blades

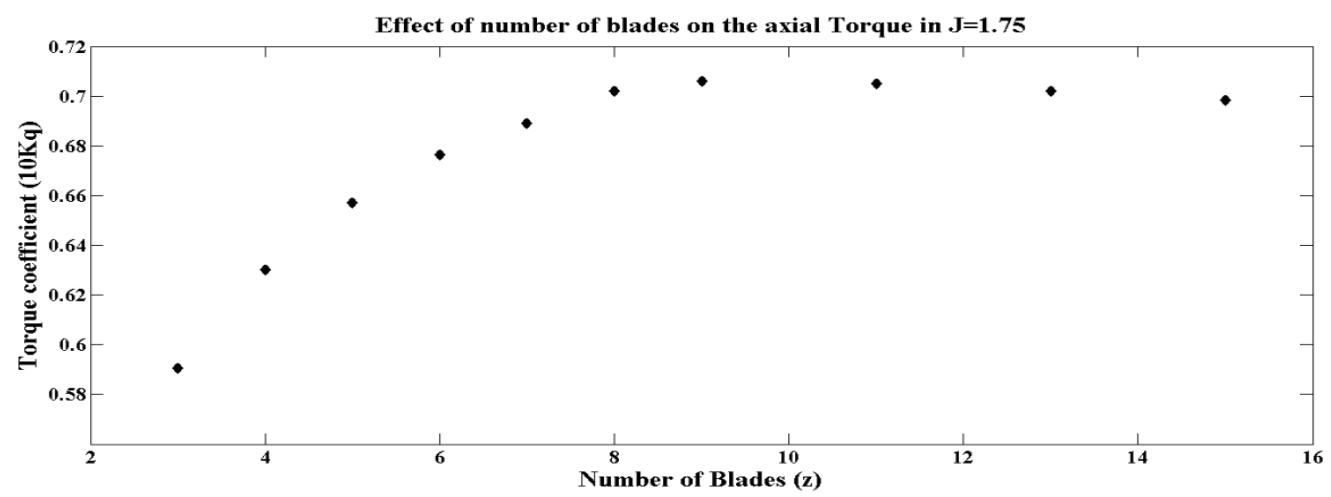

Figure 11: Magnitude of the axial torque of the propellers in the advance coefficient corresponding to the maximum efficiency versus the number of blades

As shown in Figure 8 the advance coefficients which are belong to the optimum points (maximum efficiency) are to some extent unchanged by changing the number of blades on the propellers. Figure 9 shows that the maximum efficiency of the propellers occurs in the 6-bladed propeller and has an efficiency that is about $1 \%$ higher than the 3-bladed propeller and about $4 \%$ higher than the 15-bladed propeller. The maximum efficiency changes versus the number of blades nonlinearly. But, the maximum efficiencies of the 4-, 5-, 6-, 7-, 8- and 9-bladed propellers differs each other by less than $1 \%$. The effect of number of blades on torque and thrust magnitudes at the maximum efficiency point is shown in Figures 10 and 11 respectively. Figure 10 shows that the thrust produced by the different propellers in the optimum advance coefficient augments as the number of blades increases up to nine blades (20\% increase relative to the 3-bladed propeller) and after that it incurs a small decrement towards the 15-bladed propeller ( $8 \%$ decrease relative to the 9-bladed propeller). Furthermore, it is observed that the slope of the thrust increment decreases by moving towards the 9-bladed propeller. The same behaviour is observed for the axial torque in Figure 11.

In order to investigate the effect of the number of blades on the occurrence of cavitation, the non-dimensional pressure coefficient is considered as follows:

$$
C_{P}=\frac{P-P_{\infty}}{1 / 2 \rho V^{2}}
$$

where $P$ is the static pressure, $P_{\infty}$ is the ambient pressure far from the propeller, and $V$ is the velocity of the flow far from the propeller. In this way, the minimum pressure coefficients at different radial sections of the propeller are compared (Figure 12). Figure 12 also shows the average pressure coefficient over the surfaces of the blades. To compute the pressure coefficient, it is assumed that the propellers are immersed in the water to a depth of $5 \mathrm{~m}$. 


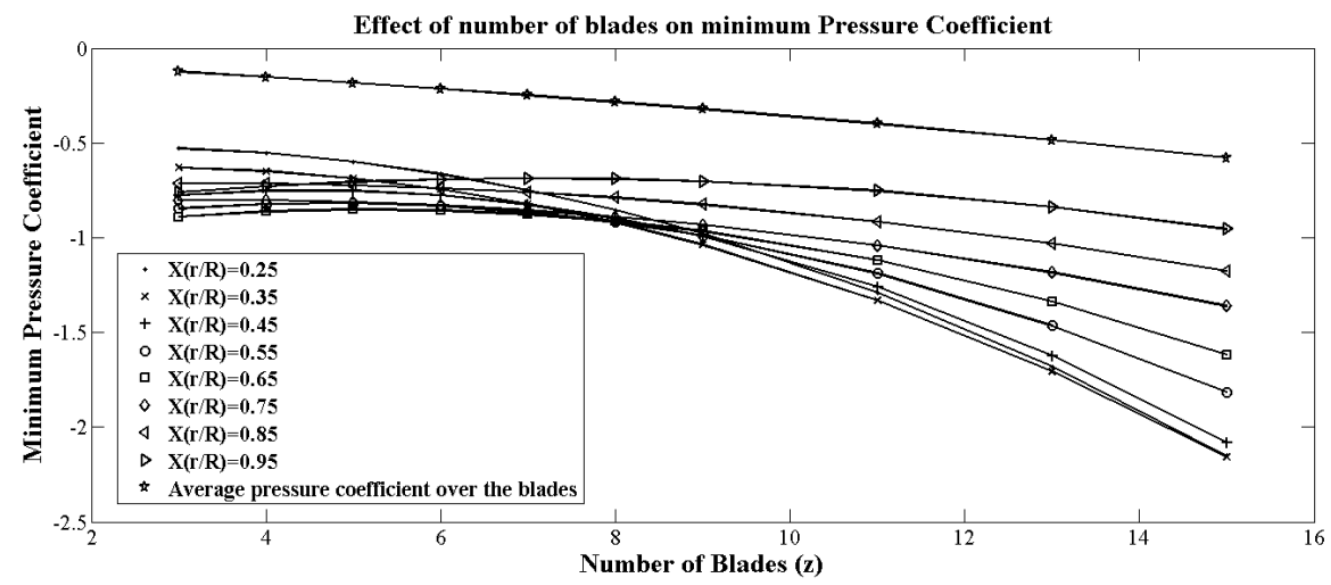

Figure 12: Comparing the minimum pressure coefficient at different radial sections between 3 - to 15 bladed propellers

Figure 12 shows that increasing the number of blades of the propellers while holding the other geometrical parameters constant decreases the magnitude of the pressure coefficient at the blades' roots significantly. This effect is observed in the radius ratios between 0.2 and 0.6. The contours of the pressure distribution over the 6- and 15-bladed propellers are presented in Figure 13 in order to make the subject clearer.

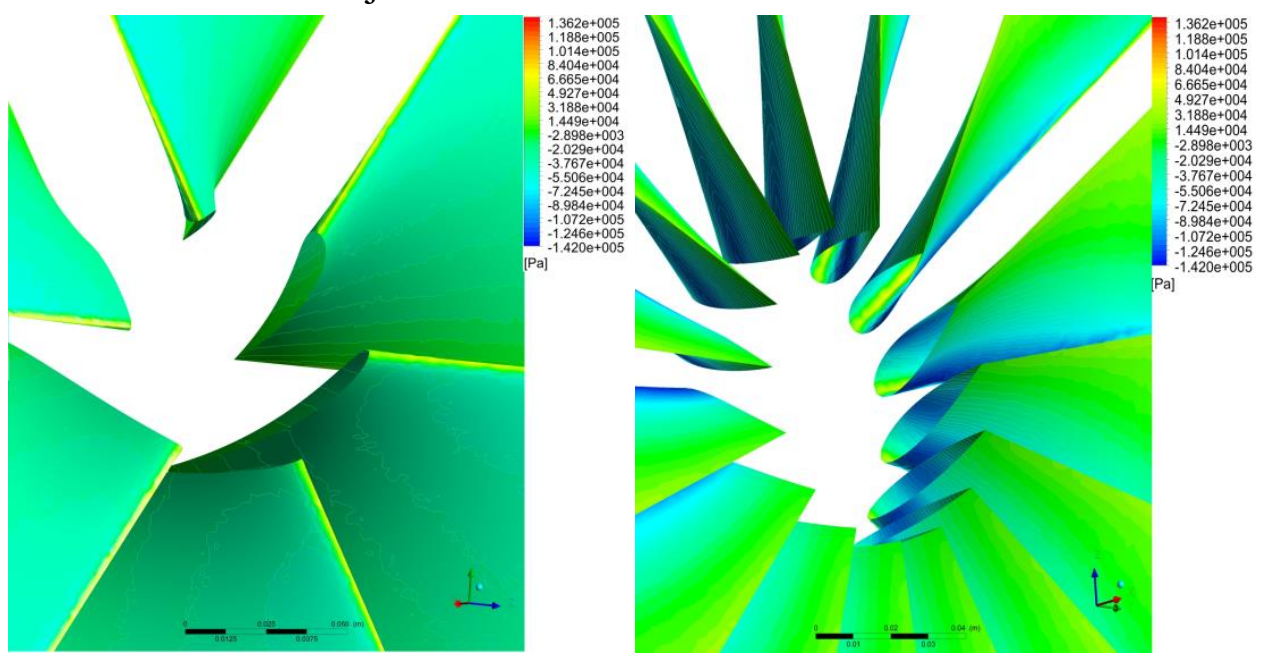

Figure 13: Contours of the pressure distribution over the: left) 6-bladed propeller; right) 15-bladed propeller

The effect of decreasing the pressure is weakened by moving from the roots of the blades to their tips. If the cavitation zone is placed in radius ratios between 0.6 and 0.7 , increasing the number of blades up to five would reinforce the blades against the cavitation occurrence slightly. While more increase in the number of blades would raise the risk of cavitation in these zones. If the cavitation zone is placed in radius ratios between 0.7 and 0.9 , increasing the number of blades up to the six do not affect the cavitation occurrence significantly. While more increase in the number of blades would raise the risk of cavitation in these zones. If the cavitation zone is placed in radius ratios between 0.9 and 1 , increasing the number of blades up to eight would reinforce the blades against the cavitation occurrence slightly. While more increase in the number of blades would raise the risk of cavitation in these zones. Figure 12 shows that the average pressure coefficient over the propeller's blades decreases significantly as the number of blades is increased. It means that the level of the pressure decrement in the roots of the propellers' blades has been severe enough which has specified the direction of the change of the averaged pressure over the blades. For the 15-bladed propeller, the magnitude of the minimum pressure coefficient is decreased by about $300 \%$ more at the propeller's root and 
by about $25 \%$ more at the propeller's tip than is the case for the 3-bladed propeller. The average magnitude of the pressure coefficient is decreased by about $360 \%$ between these two models.

Since the magnitude of the pressure distribution around the airfoil (and here around the propeller) strongly depends on the thickness ratio, it is worth to pay attention to the thickness ratio (\%) distribution over the propellers' blades which are shown in Figure 14.

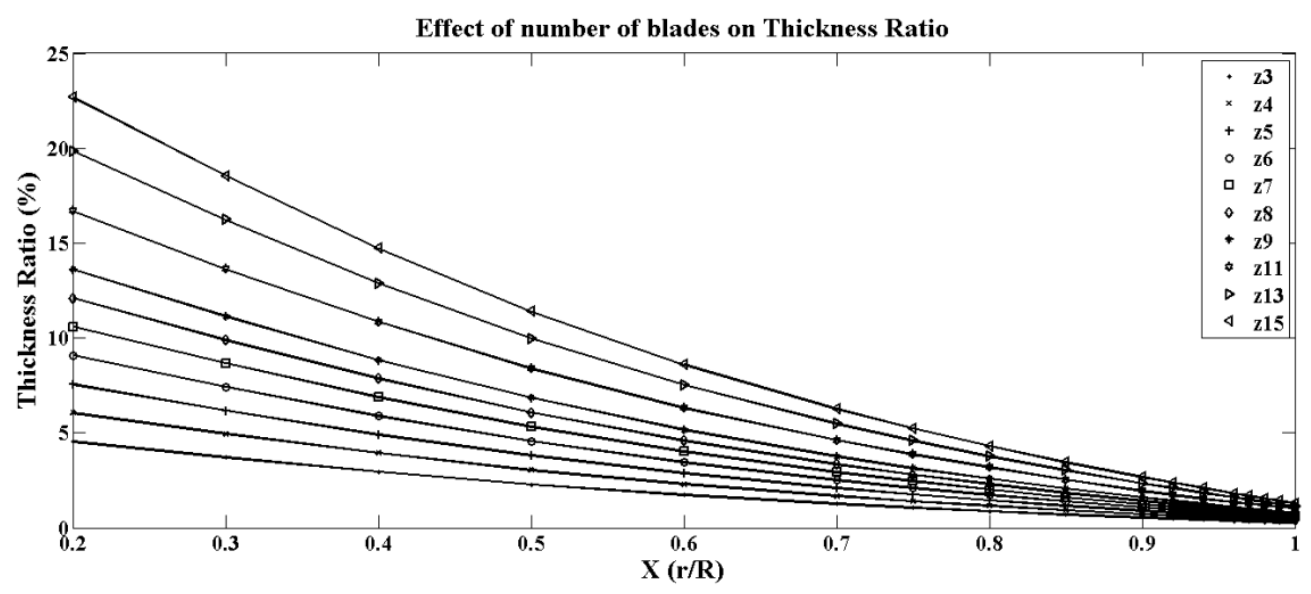

Figure 14: Comparison of the maximum thickness ratio at different radial sections between 3- to 15bladed propellers

Figure 14 shows that, the magnitude of the maximum thickness ratio of the blades was increased in every radial section by increasing the number of blades. Also, the increasing rate of the thickness ratio was constant between different radial sections of two different models. However, the magnitudes of the thickness ratio were increased significantly near the roots which have made the propellers more prone to the cavitation risk in these locations (see Figure 14).

In this research, a specified thrust of about $600 \mathrm{~N}$ is considered, and the corresponding hydrodynamic pressure is imported from the CFD solver for the corresponding FEM model. Thus, we are able to compare the structural behaviour of the propellers under the same loading condition. Since, the deflection of the propellers was very small compared to their diameters, the interaction between the fluid and solid was neglected and there was a one-time importation of the distribution of pressure from the CFD solver to the FEM solver. Figure 15 shows the results.

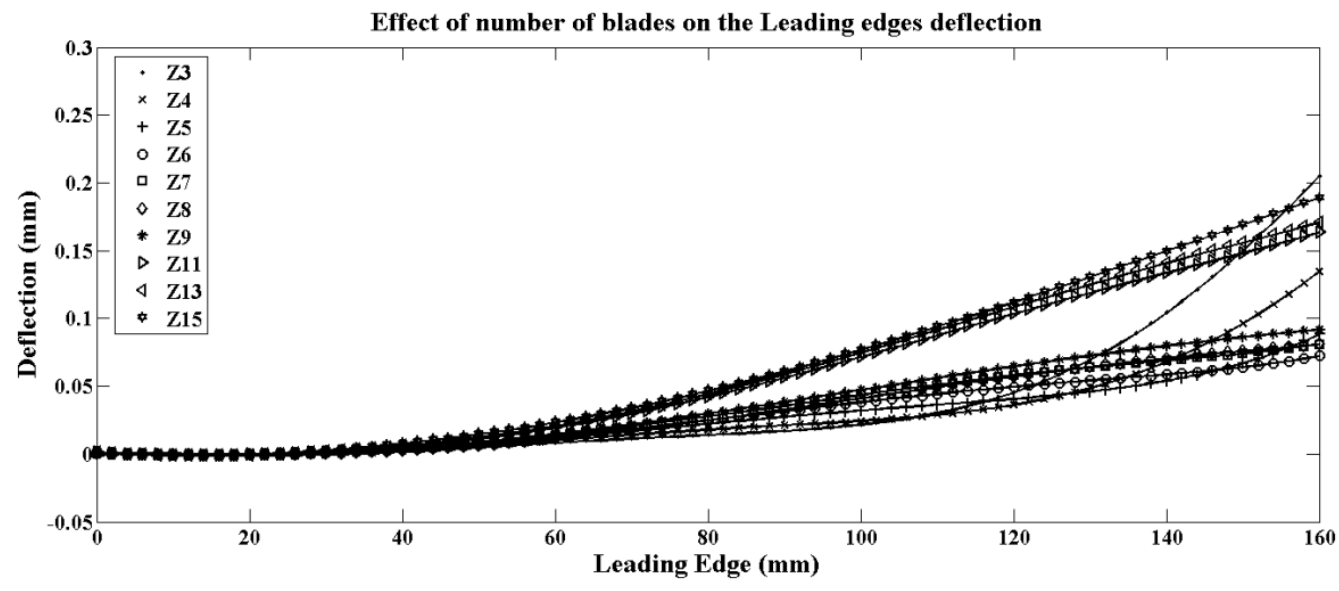

Figure 15: The maximum deflection of the propellers over their leading edges

Figure 15, shows that the magnitude of the maximum deflection which occurs over the leading edge of the propellers is reduced by increasing the number of blades up to six, but the magnitude of this deflection is increased for seven or more blades. Since, the leading edges of the propellers are rather thick, it is concluded that the number of blades has a nonlinear 
relationship with the structural deflection or the propellers' stiffness values. The maximum magnitude of the deflection is reduced by about $65 \%$ by increasing the number of blades from three to six and is reduced by about $60 \%$ by decreasing the number of blades from fifteen to six. On the other hand, Table 4 indicates that increasing the number of blades augments the maximum magnitude of the Von-Mises stresses on the roots of the propeller's blades. Figure 16 shows the distribution of the Von-Mises stresses along the mid-section of the 7-bladed and 15-bladed propellers. The reason of the difference between the overall shapes of the blades in Figure 16 is the difference between the blade numbers which has leaded to the different blade shapes. Furthermore, the difference between the legends is because of the difference between the stiffness of the blades. As it can be seen in this figure, the maximum Von-Mises stresses are happened on the roots of the blades which are found in Table 4.
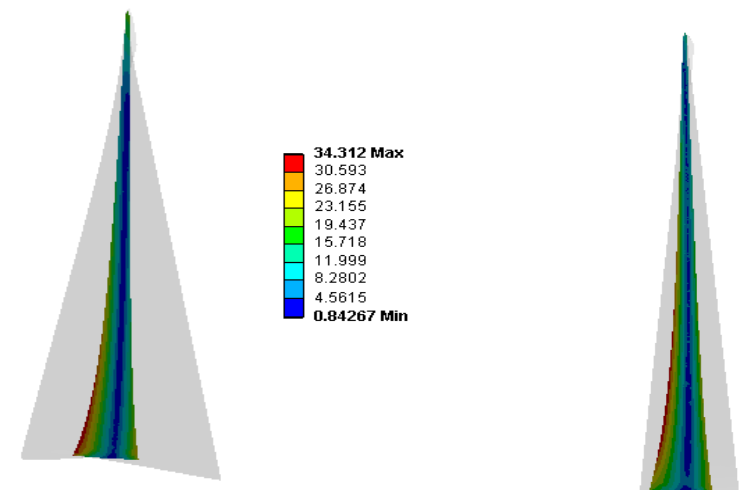

Figure 16: Distribution of the Von-Mises stresses along the mid-section of two types of propellers: left) 7-bladed propeller; right) 15-bladed propeller

Table 4: The relationship between the maximum magnitude of the Von-Mises stresses and the number of blades

\begin{tabular}{|c|c|}
\hline $\begin{array}{c}\text { Number of } \\
\text { blades }\end{array}$ & $\begin{array}{c}\text { Maximum Von-Mises } \\
\text { stress (MPa) }\end{array}$ \\
\hline 3 & 14.8 \\
\hline 4 & 20.2 \\
\hline 5 & 23.6 \\
\hline 6 & 24 \\
\hline 7 & 25.2 \\
\hline 8 & 26.2 \\
\hline 9 & 29 \\
\hline 11 & 29.8 \\
\hline 13 & 31.3 \\
\hline 15 & 34.3 \\
\hline
\end{tabular}

Table 4 indicates that the maximum magnitude of the Von-Mises stresses over the blades is increased by increasing the number of blades. Note that increasing the number of blades from 3 to 15 is increased the maximum magnitude of the Von-Mises stresses by about $130 \%$. This parameter is directly related to the structural lifetime of the propellers [16].

The data in Table 5 clearly shows that increasing the number of blades from 3 to 15 is resulted in $80 \%$ reduction in the axial length of the propellers. Figure 17 shows the axial length (the occupied axial space with the propeller) of a sample propeller. 


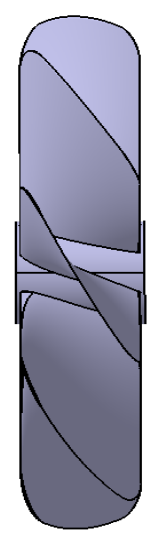

Figure 17: The side view of the 8-bladed propeller

Table 5: The relationship between the axial lengths of the propellers and the number of blades

\begin{tabular}{|c|c|}
\hline $\begin{array}{c}\text { Number of } \\
\text { blades }\end{array}$ & $\begin{array}{c}\text { Axial length of the } \\
\text { propellers (mm) }\end{array}$ \\
\hline 3 & 200 \\
\hline 4 & 150 \\
\hline 5 & 120 \\
\hline 6 & 100 \\
\hline 7 & 85.7 \\
\hline 8 & 75 \\
\hline 9 & 66.6 \\
\hline 11 & 54.3 \\
\hline 13 & 45.9 \\
\hline 15 & 39.5 \\
\hline
\end{tabular}

The obtained results could be interpreted as follows:

- Regarding the results obtained from the simulations, it is obvious that the procedure used to determine the number of blades on a propeller is a multi-objective function of efficiency, cavitation, vibrational behaviour, structural lifetime, and physical space.

- In considering cavitation, it is apparent that increasing the number of blades on a propeller changes its resistance against cavitation nonlinearly. Increasing the number of blades of the propellers is weakened them in radius ratios between 0.2 and 0.6 against the cavitation occurrence. Changing the number of blades would affect the cavitation occurrence in radius ratios between 0.6 and 1 slightly. The changes in these zones are nonlinear. Generally, it can be concluded that the propensity for a propeller to experience cavitation can be reduced by decreasing the number of blades if the cavitation zones were located closer to the roots than the tips, while the propeller's weight and its expanded area ratio are kept constant. Because increasing the thickness ratio of the blades nonlinearly changes the cavitation behaviour, it cannot be concluded that every thicker blade is more prone to the cavitation occurrence relative to a thinner one.

- Considering efficiency, the difference between propellers with three to nine blades is less than $1 \%$. However, the 15-bladed propeller has an efficiency value that is about $4 \%$ less than that of the 6-bladed propeller. The reason for this lower efficiency is increasing the magnitude of the lift-to-drag coefficient of the radial sections by increasing their thickness ratio up to a specified value. After that, the magnitude of the lift-to-drag coefficient decreases with further increase in the thickness ratio [17]. 
Thus, the maximum efficiency of the propellers is increased by increasing the number of blades from three to six, but it is decreased by any further increases in the number of blades. Also, it is observed that the advance coefficient, which is related to the maximum efficiency of the propellers, remains unchanged as the number of blades is changed. The main reason is that the radial distributions of the effective angles of attack are the same for all of the propellers. Generally, it is seen that increasing the thickness ratio of the blades changes the efficiency of the propellers nonlinearly. So, it cannot be concluded that every thicker blade has less efficiency relative to a thinner one.

- Figure 10 shows that the magnitudes of the thrust that are produced by the propellers at the optimum point are increased until the propellers have nine blades, but these values are decreased when the number of blades exceeded nine. This phenomenon occurs because of the relationship between the lift and drag coefficients and the thickness ratios. As mentioned in [17], the lift coefficient increases up to a certain value as the thickness ratio increases. If the thickness ratio continues to increase, the lift coefficient will decrease after reaching the mentioned certain value of the lift coefficient. On the other hand, the drag coefficient increases continuously as the number of blades increases. Because the thrust produced by the propeller is directly related to the lift coefficient of the radial sections and is inversely related to the drag coefficient of these sections [18], a nonlinear nature is obviously seen in its behavior.

- Figure 11 shows that the magnitude of the axial torque applied on the propeller's shaft is augmented as the number of blades is increased up to nine, after which there is a slight reduction in the torque. This event happens because the magnitude of the torque applied on the propeller's shaft is directly related to both lift and drag coefficients [18]. The lift coefficient is decreased and the drag coefficient is increased by increasing the number of blades from nine to fifteen. So, the torque is decreased, but to a far less extent than the produced thrust.

- Considering the vibration (deflection), it is observed that the structural stiffness of the propellers is nonlinearly changed by changing the number of blades. The greatest stiffness occurs in the 6-bladed propeller which is about $60 \%$ greater than that of the 3 - and 15-bladed propellers subjected to hydrodynamic pressure distributions that are corresponded to the same magnitudes of thrust.

- Considering the lifetime of the structure, it is apparent that increasing the number of blades increases the maximum Von-Mises stresses on the blades. It means that the propeller would have a shorter lifetime.

- From the geometrical point of view, it is observed that the axial lengths of the propellers are reduced by increasing the number of blades, which could be a very important issue in the case of designing the ducted propellers for underwater axisymmetric bodies.

So, it is observed that the procedure of determining the number of blades on a propeller is a multi-objective one which must be performed according to the design criteria. According to the obtained results in this research, the following flowchart (Figure 18) could be proposed in order to determine the propeller's number of blades: 


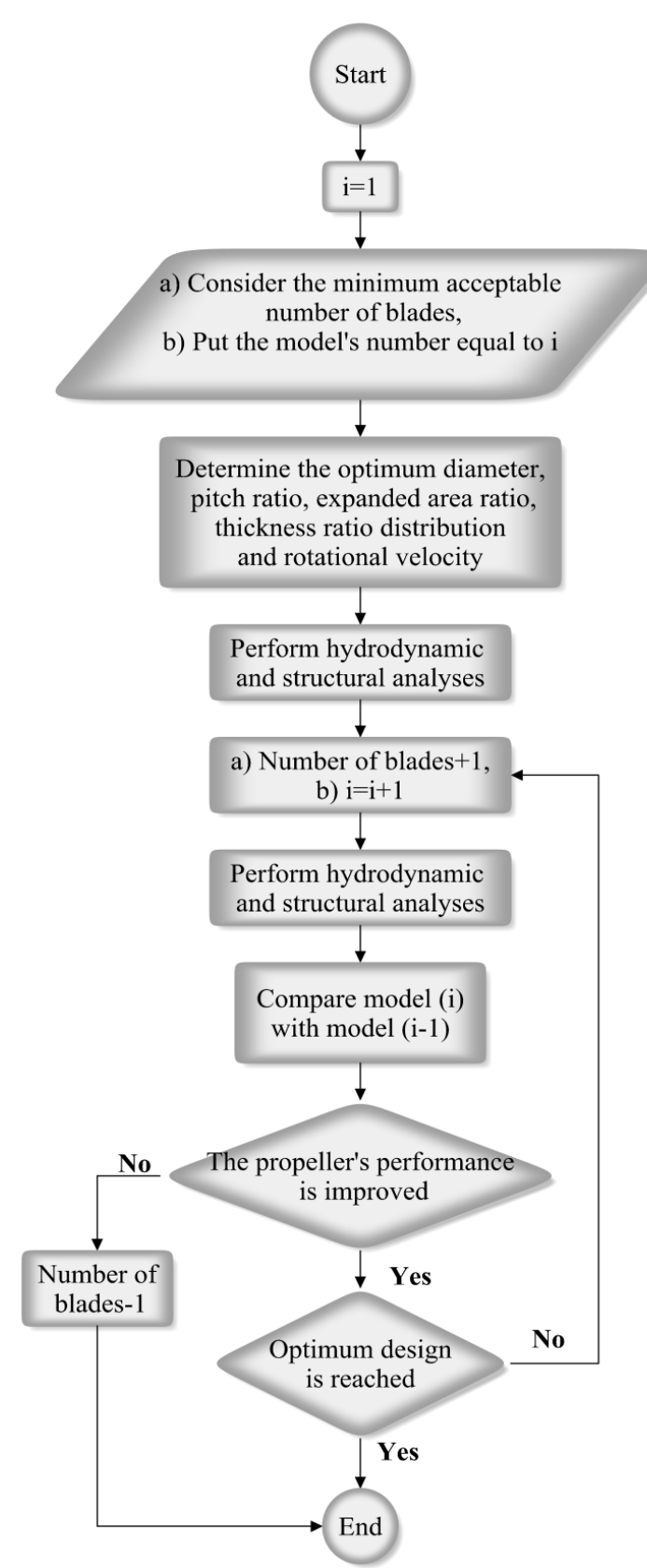

Figure 18: The determination procedure of the propeller's number of blades

At the end, it is worth to note that the Reynolds numbers which are based on the chord lengths of the propellers' blades in radius ratios equal to 0.7 have been over 1.25 million between different models in this research. It shows that the simulations have been performed in the fully turbulent flow regimes and the scale effects have been minimized as much as possible.

\section{Conclusions}

A multi-objective approach for determining the number of blades on a propeller is presented in this article. Ten different models of propellers with 4-digit NACA cross sections and the only variable being the number of blades are developed and analysed hydrodynamically and structurally. It is observed that the procedure of determining the number of blades on a propeller is a multi-objective function of hydrodynamic efficiency, cavitation risk, vibrational behaviour, structural lifetime, and physical space, which must be considered with respect to the design criteria. 


\section{Acknowledgment}

We sincerely appreciate the assistance and guidance concerning various aspects of the research that were provided by the staff of the Applied Hydrodynamics Laboratory at Iran University of Science and Technology.

\section{References}

[1] Abdel-Maksoud, M., Menter, F., \& Wuttke, H. (1998). Viscous Flow Simulations for Conventional and High-Skew Marine Propellers. Ship Technology Research 45, Schiffstechnik, 45(2), 64-71.

[2] Watanabe, T., Kawamura, T., Takekoshi, Y., Maeda, M., \& Rhee, S. H. (2003, November). Simulation of steady and unsteady cavitation on a marine propeller using a RANS CFD code. In Proceedings of the Fifth International Symposium on Cavitation, Osaka, Japan.

[3] Ghasemi, H. (2009). The effect of wake flow and skew angle on the ship propeller performance. In Scientia Iranica, 16(2), 149-158.

[4] Da-Qing, L. I. (2006). Validation of RANS predictions of open water performance of a highly skewed propeller with experiments. Journal of Hydrodynamics, Ser. B, 18(3), 520-528. http://dx.doi.org/10.1016/S1001-6058(06)60106-6.

[5] Hayati, A. N., Hashemi, S. M., \& Shams, M. (2011). A study on the effect of the rake angle on the performance of marine propellers. Proceedings of the Institution of Mechanical Engineers, Part C: Journal of Mechanical Engineering Science, 0954406211418588.

[6] Ji, B., Luo, X., Wang, X., Peng, X., Wu, Y., \& Xu, H. (2011). Unsteady numerical simulation of cavitating turbulent flow around a highly skewed model marine propeller. ASME J. Fluids Eng, 133(1), 011102. http://dx.doi.org/10.1115/1.4003355.

[7] Peng, H. H., Qiu, W., \& Ni, S. (2013). Effect of turbulence models on RANS computation of propeller vortex flow. Ocean Engineering, 72, 304-317. http://dx.doi.org/10.1016/j.oceaneng.2013.07.009.

[8] Shamsi, R., Ghassemi, H., Molyneux, D., \& Liu, P. (2014). Numerical hydrodynamic evaluation of propeller (with hub taper) and podded drive in azimuthing conditions. Ocean Engineering, 76, 121-135. http://dx.doi.org/10.1016/j.oceaneng.2013.10.009.

[9] Orszag, S. A., Yakhot, V., Flannery, W. S., Boysan, F., Choudhury, D., Maruzewski, J., \& Patel, B. (1993). Renormalization group modeling and turbulence simulations. Near-wall turbulent flows, 1031-1046.

[10] Dong, Y., Duan, X., Feng, S., \& Shao, Z. (2012). Numerical simulation of the overall flow field for underwater vehicle with pump jet thruster. Procedia Engineering, 31, 769-774. http://dx.doi.org/10.1016/j.proeng.2012.01.1100

[11] Van Doormaal, J. P., \& Raithby, G. D. (1984). Enhancements of the SIMPLE method for predicting incompressible fluid flows. Numerical heat transfer, 7(2), 147-163. http://dx.doi.org/10.1080/01495728408961817.

[12] Kaya, F., \& Karagoz, I. (2008). Performance analysis of numerical schemes in highly swirling turbulent flows in cyclones. Current science, 94(10), 1273-1278.

[13] Ghassabzadeh, M., \& Ghassemi, H. (2013). Determination of Hydrodynamics Characteristics of Marine Propeller Using Hydro-elastic Analysis. Brodogradnja, 64(1), 40-45.

[14] Carlton, J. (2011). Marine propellers and propulsion. Butterworth-Heinemann.

[15] Barnitsas, M. M., Ray, D., \& Kinley, P. (1981). KT, KQ and efficiency curves for the Wageningen B-series propellers.

[16] United States. Department of Defense. (1987). Military Standardization Handbook: Metallic Materials and Elements for Aerospace Vehicle Structures. Department of Defense.

[17] Shyy, W., Lian, Y., Tang, J., Viieru, D., \& Liu, H. (2007). Aerodynamics of low Reynolds number flyers (Vol. 22). Cambridge University Press.

[18] Benini, E. (2004). Significance of blade element theory in performance prediction of marine propellers. Ocean Engineering, 31(8), 957-974. http://dx.doi.org/10.1016/j.oceaneng.2003.12.001.

Submitted: $\quad$ 16.05.2015. $\quad$ Nowrouz Mohammad Nouri, mnouri@iust.ac.ir

Accepted: $\quad$ 23.03.2016. $\quad$ School of Mechanical Engineering, Iran University of Science and Technology, Tehran, Iran 